\title{
REFLECTION POSITIVITY FOR UNITARY REPRESENTATIONS OF LIE GROUPS
}

\author{
HUMBERTO PRADO
}

(Communicated by Palle E. T. Jorgensen)

\begin{abstract}
Let $G$ be a Lie group, and let $\sigma$ be an involutive automorphism on $G$. Then we establish a correspondence between unitary representations of $G$ and unitary representations of a simply connected Lie group $G^{*}$ dual to $G$, where the duality is defined by the given involution $\sigma$. The correspondence is obtained from a geometric assumption that was considered earlier in connection with reflection positivity. As a consequence of this construction, we obtain unitary representations of universal covering groups.
\end{abstract}

\section{INTRODUCTION}

The notion of representing Lie subsemigroups by contraction operators in Hilbert space arose first in relativistic quantum field theory as a tool in the geometric construction of strongly continuous unitary representations of the universal covering group $G^{*}$ for the Minkowkian conformal group $\mathrm{SO}(4,2) / \mathbb{Z}_{2}$, [LM]. The first instance of this construction was to represent a certain subsemigroup $\Gamma$ of the Euclidean conformal group $\mathrm{S}_{0} \mathrm{O}(5,1)$, by contractions $T_{\Lambda}$, $\Lambda \in \Gamma$, acting on a given Hilbert space $\mathscr{H}$. Lüsher and Mack [LM] showed (by analytic continuation of the group parameters) that the operators $T_{\Lambda}, \Lambda \in \Gamma$, give rise to a strongly continuous, unitary representation of the simply connected Lie group $G^{*}$ on the space $\mathscr{H}$, thus providing a noncommutative generalization of the Hille-Yosida theorem for Lie subsemigroups.

The mathematical problem that was left open was stated in the general context of virtual representations, [FOS, Se] or local representations of symmetric spaces $[\mathrm{J} 1, \mathrm{~J} 2]$. These representations were studied in particular cases, first by Klein and Landau [KL], and independently also by Fröhlich, Osterwalder, and Seiler [FOS]. The framework given in the earlier papers was a symmetric space $(G, K, \sigma)$, a simply connected Lie group $G^{*}$ dual to $G$ under the Weyl unitary trick, a Hilbert space $\mathscr{H}$, and a local homomorphism $\rho$ from a neighborhood of the identity of $G$ into densely defined operators in $\mathscr{H}$. Then it

Received by the editors September 10, 1990.

1980 Mathematics Subject Classification (1985 Revision). Primary 46N05, 47D05, 47A67, 22E45.

Key words and phrases. Local representations, symmetric spaces, reflection positivity, induced representations and phrases. 
was shown [FOS] (for a very special class of symmetric spaces and under a restrictive spectral assumption), that the given local representation $\rho$ implements a unitary representation of the group $G^{*}$ on the same Hilbert space $\mathscr{H}$. The problem has been solved in its full generality by Jorgensen [J1, J2] where the above-mentioned restrictive assumptions are entirely removed.

In this paper we give a geometric construction of local representations of a given symmetric space. One of our basic assumptions relies on reflection positivity (alias reflection positivity systems). This concept is taken from quantum field theory, and it was introduced by Osterwalder and Schrader [OS] in a construction of a geometric correspondence between the Euclidean axioms and the relativistic axioms.

In the general context of group representations, reflection positivity allows one to obtain contractive representations from unitary representations [LM, S]. We have adapted this viewpoint in order to produce local representations of general symmetric spaces $(G, K, \sigma)$ from given unitary representations of the group $G$.

We first suppose that, given at the outset, there is a Lie group $G$ together with an involutory automorphism $\sigma$. Let $(G, K, \sigma)$ be a corresponding symmetric space with a choice of subgroup $K$, see [H]. Let $\tau$ be a unitary representation of $G$, which is induced from a given representation $L$ of some closed subgroup $H$ of $G$. Let $\mathscr{H}^{L}(G)$ be the Hilbert space for the action $\tau$. We assume further that the Hilbert space $\mathscr{H}^{L}(G)$ comes equipped with a reflection positivity system (a notion, which is introduced in $\S 2$ ). For such a representation $\tau$ we show, under mild assumptions, that we can associate a local representation $\rho$ of $(G, K, \sigma)$ on some new Hilbert space $\mathscr{H}_{0}$ obtained by a quotient construction in a subspace of $\mathscr{H}^{L}(G)$. We then make an analytic continuation of $\rho$ to obtain a new unitary representation $\pi$ of a Lie group $G^{*}$, which is dual to $G$ (where the duality is defined from the given involution $\sigma$ ). Moreover, the Hilbert space of the unitary representation $\pi$ of $G^{*}$ is $\mathscr{H}_{0}$. A special case of this construction yields a family of unitary representations studied in earlier papers on quantum field theory, see e.g. [Se].

Our results generalize the main theorems of [FOS] and [S] respectively, by allowing us to obtain and identify local representations that can be analytically continued to unitary representations of the group $G^{*}$. Moreover, this method applies to a wide range of symmetric spaces and unitary representations $\tau$. We also note that the example given by the above authors fall into the general setting dictated by $\S \S 3,4$.

The paper is organized as follows: In $\S 2$ we introduce the basic ingredients. We also present the definition of local representations for symmetric spaces. In $\S 3$ we give the hypotheses that are necessary to produce local representations. The main theorems are in $\S 4$.

We assume, without mention, that all the group representations are strongly continuous.

\section{Preliminaries}

2.1. A reflection positivity system consists of a triple $(\mathscr{H}, P, \theta)$ where $\mathscr{H}$ is a Hilbert space, $P$ is an orthogonal projection on $\mathscr{H}$, and $\theta$ is an involution 
such that

$$
P \theta P \geq 0 \text {. }
$$

For vectors $f, h \in \operatorname{Ran} P$, define

$$
\langle f, h\rangle_{\theta}:=\langle f, \theta h\rangle,
$$

where the right-hand side is the given inner product of $\mathscr{H}$. It follows that (2.2) is a semi-inner product on the range of $P$. Let $N$ be the null space of $\langle,\rangle_{\theta}$. We define $\mathscr{H}_{0}$ to be the Hilbert space that is obtained by completing the quotient $\operatorname{Ran} P / N$ with respect to the norm induced by $\langle,\rangle_{\theta}$. We use the same symbol to denote the scalar product induced by $\langle,\rangle_{\theta}$ on $\mathscr{H}_{0}$.

Let $\varphi$ be the canonical map from $\operatorname{Ran} P$ onto $\mathscr{H}_{0}$. Since $\theta \geq 0$ on $\operatorname{Ran} P$, the restriction $\theta \uparrow \operatorname{Ran} P$ is a selfadjoint operator. It follows from Cauchy Schwarz that

$$
\langle\varphi(f), \varphi(f)\rangle_{\theta} \leq\|f\|^{2}
$$

for all $f \in \operatorname{Ran} P$.

2.2. A symmetric space is defined to be a triple $(G, K, \sigma)$, where $G$ is a Lie group, $K$ is a closed subgroup, and $\sigma$ is an involutive automorphism of $G$ with $K$ as fixed point subgroup. Let $\mathfrak{g}$ be the Lie algebra of $G$, and let $\mathfrak{g}=\mathfrak{k} \oplus m$ be the corresponding decomposition of $\mathfrak{g}$, as real vector space, into eigenspaces for the differential of $\sigma$, where $k$ corresponds to the Lie algebra of the subgroup $K$. The dual symmetric Lie algebra is defined by $\mathfrak{g}^{*}=\mathfrak{k} \oplus i m$, and $\mathfrak{g}^{*}$ is again a real Lie algebra. We define $G^{*}$ to be the simply connected Lie group with Lie algebra $\mathfrak{g}^{*}$.

2.3. Let $(G, K, \sigma)$ be a symmetric space. Then a local representation of $(G, K, \sigma)$ is defined to be a system $(\mathscr{H}, \mathscr{D}, U, \rho)$, where $\mathscr{H}$ is a Hilbert space, $\mathscr{D}$ is a dense linear subspace of $\mathscr{H}$, and $\rho$ is a local homomorphism from the neighborhood $U$ of the identity in $G$ to unbounded operator on $\mathscr{H}$, such that:

(L.1) The restriction of $\left.\rho\right|_{K \cap U}$ is defined for all $k$ in $K$, and extends to strongly continuous unitary representation of $K$ on $\mathscr{H}$.

(L.2) For all $g$ in $U$, the space $\mathscr{D}$ is contained in the domain $D\left(\rho_{g}\right)$ of $\rho_{g}$.

(L.3) Given $g_{1}, g_{2}$, the product $g_{1} g_{2}$ in $U$, and $f$ in $\mathscr{D}$, the vector $\rho_{g_{2}}(f)$ is assumed to belong to $D\left(\rho_{g_{1}}\right)$ and

$$
\rho_{g_{1}}\left(\rho_{g_{2}}(f)\right)=\rho_{g_{1} g_{2}}(f) .
$$

(L.4) For all $y \in m$ and $t \in \mathbb{R}, t$ in a small neighborhood centered at 0 such that $\exp (t y) \in U$, the operators $\rho_{\exp (t y)}$ are assumed hermitian, defined on $\mathscr{D}$, and satisfying

$$
\lim _{t \rightarrow 0} \rho_{\exp (t y)}(f)=f
$$

for all $f \in \mathscr{D}, y \in m$.

(L.5) For all $y \in m, f \in \mathscr{D}$, the function $k \rightarrow \rho_{\exp \left(\operatorname{Ad}_{k}(y)\right)}(f)$ is locally integrable on the following subset of $K$

$$
\left\{k \in K: \exp \left(\operatorname{Ad}_{k}(y)\right) \in U\right\} \text {. }
$$


Remark 2.1. An earlier definition given by [FOS] assumes that the subset $m_{U}$ of $m$, defined by

$$
m_{U}=\{y \in m: \exp (y) \in U\},
$$

is star shaped. We note that this assumption can be removed from the hypothesis. This follows from the fact that for arbitrary $y \in m$, there is $t_{y}>0$ such that $\exp \left(t_{y}\right) \in U$ for all $|t| \leq t_{y}$. Thus $\rho_{\exp (t y)}$ is well defined for all $t,|t| \leq t_{y}$, and (L.4) is still satisfied.

\section{LOCAL AXIOMS}

Let $(G, K, \sigma)$ be a symmetric space. Suppose that $H$ is a closed subgroup of $G$ such that

$$
\sigma(h) \in H \quad \text { for all } h \in H .
$$

Let $L$ be a representation of $H$ on a Hilbert space $\mathscr{Z}$ ( $L$ not necessarily unitary), such that

$$
L_{\sigma(h)}=L_{h} \quad \text { for all } h \in H .
$$

We denote by $\tau$ the representation of $G$, induced from the representation $L$ of $H$, and recall that $\tau$ acts on a Hilbert space $\mathscr{H}^{L}(G)$ of functions $f$ from $G$ to $\mathscr{Z}$, for which

$$
f(g h)=L_{h^{-1}}(f(g)) \quad \text { for all } g \in G, h \in H,
$$

and such that the function $g \rightarrow\langle f(g), v\rangle$ is measurable for all $v \in \mathscr{Z}$. The action $\tau$ on $\mathscr{H}^{L}(G)$ is then given by

$$
\tau_{g}(f)\left(g_{0}\right)=f\left(g^{-1} g_{0}\right), \quad g, g_{0} \in G .
$$

Now we state the following axioms for symmetric spaces $(G, K, \sigma)$ satisfying (3.1), and induced representations $\tau$ for which (3.2) holds.

Let $U$ be a neighborhood of the identity in $G$. Let $C_{+}$and $V$ be given open subsets of $G$ such that $V \subset C_{+}$. Then assume:

(i) $k C_{+} \subseteq C_{+}$, for all $k \in K$;

(ii) $g V \subseteq C_{+}$, for all $g \in U$.

(A.2) There is an element $y \in m$ such that

(i) $\exp (t y) C_{+} \subseteq C_{+}$for all $t \geq 0$;

(ii) for every compact subset $C$ of $C_{+}$, there is $t_{0}>0$ such that $\exp (t y) C \subset V$ for all $t \geq t_{0}$.

(A.3) For $f \in \mathscr{H}^{L}(G)$, define the linear involution

$$
\theta(f)(g)=f(\sigma(g)) \text { for all } g \in G .
$$

Let $P$ be the orthogonal projection of $\mathscr{H}^{L}(G)$ onto $\operatorname{Ran} P=\operatorname{clo}\{f: \operatorname{supp} f \subset$ $\left.C_{+}\right\}$. Then it is assumed that $\mathscr{H}^{L}(G)$ carries an inner product such that the triple $\left(\mathscr{C}^{L}(G), P, \theta\right)$ is a reflection positivity system and such that $\tau$ is a unitary representation of $G$ on $\mathscr{H}^{L}(G)$.

Remarks 3.1. We note that requirement (A3) forces us to consider some inner product on $\mathscr{H}^{L}(G)$ that might be different from the original scalar product obtained by the induction machinery. 
3.2. Throughout the rest of the paper we assume that the neighborhood $U$, satisfying (A1), is chosen symmetric. We further note that $U$ could be chosen to be invariant under the involutive automorphism $\sigma$ of the group $G$. If not, we could work with a smaller neighborhood $U^{\prime}$, given by $U^{\prime}=U \cap \sigma(U)$. Henceforth, we assume that the neighborhood $U$ is $\sigma$-invariant and symmetric.

\section{MAIN Results}

Throughout this section all the symmetric spaces and representations are assumed to satisfy the properties from $\S 3$.

The following elementary lemma is useful in the proof of our main result.

Lemma 4.1. Let $(G, K, \sigma)$ be a symmetric space with $\tau, L$, and $\theta$ as specified. Then

$$
\theta \tau_{g}=\tau_{\sigma(g)} \theta \quad \text { for all } g \in G .
$$

Proof. Since $L_{\sigma(h)}=L_{h}$ for all $h \in H$, thus $\theta(f) \in \mathscr{H}^{L}(G)$ for all $f \in$ $\mathscr{H}^{L}(G)$, and equation (4.1) follows from (3.3).

Theorem 4.2. Let $(G, K, \sigma)$ be a symmetric space, and $H$ a subgroup of $G$. Let $\tau$ be an induced representation of $G$, from a representation $L$ of $H$. If (A1)-(A3) are satisfied, then the unitary representation $\tau$ gives rise to a local representation of the symmetric space $(G, K, \sigma)$.

We show that the axioms (A1)-(A3) imply the defining properties (L1)-(L5) of a local representation. During the course of the proof we obtain a Hilbert space $\mathscr{H}_{0}$ from reflection positivity in $\mathscr{H}^{L}(G)$, a dense linear subspace $\mathscr{D}$, and a local homomorphism $\rho$, defined on the neighborhood $U$ (specified in (A1)), such that the quadruple $(\mathscr{H}, \mathscr{D}, U, \rho)$ is a local representation of $(G, K, \sigma)$.

Proof of Theorem 4.2. Step 1. The Hilbert space. For arbitrary $\mathscr{O}$ open subset of $C_{+}$, define $\mathscr{S}(\mathscr{O})$ to be the space of all those $f \in \operatorname{Ran} P$ such that $\operatorname{supp} f$ is compact and contained in $\mathscr{O}$. Thus

$$
\mathscr{S}(V) \subset \mathscr{S}(\mathscr{O}) \subset \operatorname{Ran} P .
$$

Then we define, for $f, h \in \mathscr{S}\left(C_{+}\right)$,

$$
\langle f, h\rangle_{\theta}:=\langle f, \theta h\rangle .
$$

It follows from (A3) that $\langle,\rangle_{\theta}$ is a semi-inner product on $\mathscr{S}\left(C_{+}\right)$. Let $N$ be the null space of $\langle,\rangle_{\theta}$. We define $\mathscr{H}_{0}$ to be the Hilbert space that is obtained by completing $\mathscr{S}\left(C_{+}\right) / N$ with respect to the norm induced by $\langle$,$\rangle .$ Let $\varphi$ be the canonical map from $\mathscr{H}\left(C_{+}\right)$into $\mathscr{H}_{0}$, and let $\langle,\rangle_{\theta}$ be the scalar product of $\mathscr{H}_{0}$ inherited from $\langle,\rangle_{\theta}$.

Step 2. The dense linear subspace $\mathscr{D}$. We show that $\mathscr{D}:=\varphi(\mathscr{S}(V))$ is a dense subspace of $\mathscr{H}_{0}$.

Recall that $\sigma(\exp (y))=\exp (-y)$ for every $y \in m$; this fact, together with Lemma (4.1), imply

$$
\tau_{\exp (-y)} \theta=\theta \tau_{\exp (y)} .
$$

For arbitrary $f$ in $\mathscr{S}\left(C_{+}\right)$and $t \geq 0$, define the operator

$$
S_{t}(f):=\tau_{\exp (t y)}(f),
$$


where $y \in m$ satisfies the properties stated in (A2). We next show that $\left\{S_{t}\right\}_{t>0}$ induces a strongly continuous symmetric semigroup on the subspace $\varphi\left(\mathscr{S}\left(C_{+}\right)\right)$, which by construction is dense in the Hilbert space $\mathscr{H}_{0}$.

Let $f$ be in $\mathscr{S}\left(C_{+}\right)$, then from relation (A2)(i), it follows that

$$
\exp (t y) \operatorname{supp} f \subset C_{+}, \quad t \geq 0 .
$$

Furthermore, $\operatorname{supp} S_{t}(f)=\exp (t y) \operatorname{supp} f$. Thus

$$
S_{t}\left(\mathscr{S}\left(C_{+}\right)\right) \subseteq \mathscr{S}\left(C_{+}\right), \quad t \geq 0 .
$$

Equation (4.3) and the unitarity of the operators $\tau_{\exp (t y)}$ imply

$$
\left\langle S_{t}(f), f\right\rangle_{\theta}=\left\langle f, S_{t}(f)\right\rangle_{\theta}
$$

for all $f \in \mathscr{S}\left(C_{+}\right), t \geq 0$. Hence

$$
\begin{aligned}
\left\langle S_{t}(f), S_{t}(f)\right\rangle_{\theta} & =\left\langle f, S_{2 t}(f)\right\rangle_{\theta} \\
& \leq\langle f, f\rangle_{\theta}^{1 / 2}\left\langle S_{2 t}(f), S_{2 t}(f)\right\rangle_{\theta}^{1 / 2},
\end{aligned}
$$

so $S_{t}(N) \subset N$ for $t \geq 0$.

Because $\tau_{\exp (t y)}$ is strongly continuous, we have the estimate

$$
\begin{aligned}
\left\langle S_{t}(f)-f, S_{t}(f)-f\right\rangle_{\theta}= & \left\langle S_{t}(f)-f, \theta S_{t}(f)-\theta(f)\right\rangle \\
\leq & \left\langle S_{t}(f)-f, S_{t}(f)-f\right\rangle^{1 / 2} \\
& \times\left\langle\theta S_{t}(f)-\theta(f), \theta S_{t}(f)-\theta(f)\right\rangle^{1 / 2} \\
= & \left\|\tau_{\exp (t y)}(f)-f\right\|\left\|\tau_{\exp (-t y)} \theta(f)-\theta f\right\| .
\end{aligned}
$$

It follows that $\left\langle S_{t}(f)-f, S_{t}(f)-f\right\rangle_{\theta}$ tends to 0 as $t \rightarrow 0^{+}$.

The homomorphism property of $\tau_{\exp (t y)}$ implies that if $t, t^{\prime}$ are given positive, then

$$
S_{t} S_{t^{\prime}}=S_{t+t^{\prime}}
$$

We are now ready to construct a strongly continuous semigroup $\left(T_{t}\right)_{t \geq 0}$ on $\varphi\left(\mathscr{S}\left(C_{+}\right)\right)$. For $f \in \mathscr{S}\left(C_{+}\right)$, define

$$
T_{t}(\varphi(f)):=\varphi\left(S_{t}(f)\right), \quad t \geq 0 .
$$

Let $f \in \mathscr{S}\left(C_{+}\right)$. Then applying (A3)(ii), we get the existence of some $t_{f}>0$ such that

$$
\exp (t y) \operatorname{supp} f \subset V \text { for all } t \geq t_{f} .
$$

Thus if $t \geq t_{f}$, then $\operatorname{supp} S_{t}(f) \subset V$. Hence $T_{t}(\varphi(f)) \in \mathscr{D}$ for all $t \geq t_{f}$. Therefore, by applying the results given in [P2], $\mathscr{D}$ is dense in $\mathscr{H}_{0}$.

Step 3. The local homomorphism. Let $f, h$ be in $\mathscr{S}(V)$. Then (4.1) implies that

$$
\left\langle\tau_{g}(f), h\right\rangle_{\theta}=\left\langle f, \tau_{\sigma(g-1)}(h)\right\rangle_{\theta}
$$

for all $g \in U$.

Let $f \in N \cap \mathscr{S}(V)$ and $h$ in $\mathscr{S}(V)$. Then for arbitrary $g \in U$, we have

$$
\begin{aligned}
\left|\left\langle\tau_{g}(f), h\right\rangle_{\theta}\right| & =\left|\left\langle f, \tau_{\sigma(g-1)}(h)\right\rangle_{\theta}\right| \\
& \leq\langle f, f\rangle_{\theta}^{1 / 2}\left\langle\tau_{\sigma(g-1)}(h), \tau_{\sigma(g-1)}(h)\right\rangle_{\theta}^{1 / 2} .
\end{aligned}
$$


So $\tau_{g}(N \cap \mathscr{S}(V)) \subset N$. We, therefore, can define

$$
\rho_{g}(\varphi(f)):=\varphi\left(\tau_{g}(f)\right)
$$

for $g \in U, f \in \mathscr{S}(V)$. Thus for each $g \in U, \rho_{g}$ is an operator with domain $D_{g}$ and $\mathscr{D} \subset D_{g}$.

Let $f \in \mathscr{S}(V), g \in U$. Then

$$
\operatorname{supp} \tau_{g}(f)=g \operatorname{supp} f .
$$

Thus if $g_{1}, g_{2}$, and $g_{1} g_{2}$ are in $U$ and $f \in \mathscr{S}(V)$, we have

$$
g_{1} g_{2} \operatorname{supp} f \subset C_{+}
$$

and

$$
\operatorname{supp} \tau_{g_{1}}\left(\tau_{g_{2}}(f)\right)=g_{1} g_{2} \operatorname{supp} f
$$

so $\rho_{g_{2}}(f)$ belongs to $D_{g_{1}}$ and

$$
\rho_{g_{1}} \rho_{g_{2}}(\varphi(f))=\rho_{g_{1} g_{2}}(\varphi(f)) .
$$

Step 4. We now show that (L.4) and (L.5) of $\S 2$ are satisfied. Let $y \in m$ and let $t$ be sufficiently small such that $\exp (t y) \in U$. Then

$$
\left\langle\tau_{\exp (t y)}(f), f\right\rangle_{\theta}=\left\langle f, \tau_{\exp (t y)}(f)\right\rangle_{\theta}
$$

for all $f \in \mathscr{S}(V)$. We see that the operators $\rho_{\exp (t y)}$ are hermitian and defined on $\mathscr{D}$. Moreover, the strong continuity of $g \rightarrow \tau_{g}(f)$, for arbitrary $f \in$ $\mathscr{H}^{L}(G)$, implies

$$
\lim _{t \rightarrow 0} \rho_{\exp (t y)}(\varphi(f))=\varphi(f)
$$

for all $y \in m, \varphi(f) \in \mathscr{D}$. Furthermore, for arbitrary $y \in m, \varphi(f) \in \mathscr{D}$, the function $k \rightarrow \rho_{\exp \left(\operatorname{Ad}_{k}(y)\right)}(\varphi(f)), k \in K$, is locally integrable over every compact subset of $\left\{k \in K: \exp \left(\operatorname{Ad}_{k}(y)\right) \in U\right\}$.

Since $k C_{+} \subset C_{+}$for all $k \in K, \tau_{k}\left(\mathscr{S}\left(C_{+}\right)\right) \subseteq \mathscr{S}\left(C_{+}\right)$for all $k \in K$. Because $\sigma(k)=k$, we have

$$
\left\langle\tau_{k}(f), \tau_{k}(f)\right\rangle_{\theta}=\langle f, f\rangle_{\theta}, \quad f \in \mathscr{S}\left(C_{+}\right)
$$

and

$$
\rho_{k}(\varphi(f))=\varphi\left(\tau_{k}(f)\right), \quad k \in U \cap K,
$$

$\varphi(f) \in \mathscr{D}$. It follows that $\rho$ extends to a unitary representation of the subgroup $K$ on the Hilbert space $\mathscr{H}_{0}$.

Remark 4.3. If the neighborhood $U$ of the identity in $G$, specified by (A1), turns out to be invariant under right translation by elements of $K$, then the quadruple $\left(\mathscr{H}_{0}, \mathscr{D}, U, \rho\right)$ that was obtained from the last theorem satifies (L1)-(L5) of $\S 2$. Hence we have all the hypothesis of Theorem 1 of [J2].

Thus we have:

Corollary 4.4. Suppose the neighborhood $U$, given in (A1), is invariant under right translations by elements of the subgroup $K$. Then the local representation $\left(\mathscr{K}_{0}, \mathscr{D}, U, \rho\right)$ can be analytically continued to a strongly continuous unitary representation of the simple connected Lie group $G^{*}$ on the Hilbert space $\mathscr{H}_{0}$.

Remark 4.5. Since $\theta^{2}=I$, then $\theta$ is a selfadjoint operator on the Hilbert $\mathscr{H}^{L}(G)$. Using Cauchy-Schwarz, we note that the operators $\left(S_{t}\right)_{t \geq 0}$, defined by equation (4.4), verify the following estimates (considered earlier, e.g. [OS]). 
For arbitrary $f \in \mathscr{S}\left(C_{+}\right)$and $t \geq 0$, we have

$$
0 \leq\left\langle f, S_{2 t}(f)\right\rangle_{\theta} \leq\|f\|^{2},
$$

where the right-hand side is the norm of $\mathscr{H}^{L}(G)$. Moreover, for every positive integer $n$ we have

$$
\begin{aligned}
\left\langle S_{t}(f), S_{t}(f)\right\rangle_{\theta} & \leq\langle f, f\rangle_{\theta}^{1 / 2}\left\langle f, S_{4 t}(f)\right\rangle_{\theta}^{1 / 2} \leq \cdots \\
& \leq\langle f, f\rangle_{\theta}^{1-1 / 2 n}\left\langle f, S_{2^{n+1} t}(f)\right\rangle_{\theta}^{1 / 2 n} \\
& \leq\langle f, f\rangle_{\theta}^{1-1 / 2 n}\|f\|^{1 / 2^{n-1}} .
\end{aligned}
$$

Then taking the limit as $n \rightarrow+\infty$, we get

$$
\left\langle S_{t}(f), S_{t}(f)\right\rangle_{\theta} \leq\langle f, f\rangle_{\theta} .
$$

Hence for $f \in \mathscr{S}\left(C_{+}\right)$and $t \geq 0$, we get

$$
\left\|T_{t}(\varphi(f))\right\|_{\theta} \leq\|\varphi(f)\|_{\theta},
$$

which by the spectral theorem, implies that the selfadjoint infinitesimal generator of $T_{t}, t \geq 0$ is positive.

4.6. In order to remove the invariance condition imposed on the neighborhood $U$ (given by Corollary 4.4), we assume extra hypothesis on the representation $\tau$. These assumptions are similar to those stated in [J1].

We suppose that the axioms (A1)-(A3) hold for the representation $\tau$. Then we add the following:

(A4) $d \tau(y)(f) \in \mathscr{S}(V)$, for all $f \in \mathscr{S}(V), y \in m$.

(A5) For each $f \in \mathscr{S}(V)$, there is a neighborhood $V_{f}$ of the identity in $K$ such that

(i) $U V_{f} \subset U^{2}$ and

(ii) $\tau_{k}(f) \in \mathscr{S}(V)$, for all $k \in V_{f}$.

The following version of Corollary 4.4 is obtained.

Theorem 4.7. Let $(G, K, \sigma)$ be a symmetric space such that (A1)-(A5) hold. Then the unitary representation $\tau$ of $G$ induces a local representation $\rho$ of $(G, K, \sigma)$. Moreover, $\rho$ can be analytically continued to a unitary representation of the simply connected Lie group $G^{*}$.

Proof. Since (A1)-(A3) hold, it follows from Theorem 4.2, that there is a local representation $\left(\mathscr{H}_{0}, \mathscr{D}, U, \rho\right)$ of $(G, K, \sigma)$ associated to the given representation $\tau$. We show that (A4), (A5) above imply that $\rho$ satisfies the assumptions of Theorem 4.5, c.f. [J1]. Notice that

$$
\theta d \tau(y)=-d \tau(y) \theta
$$

for all $y \in m$.

Also we assumed

$$
d \tau(y)(\mathscr{S}(V)) \subseteq \mathscr{S}(V), \quad y \in m .
$$

Then from the unitarity of the operators $\tau_{g}, g \in G$, we have for arbitrary $f, h$ in $\mathscr{S}(V), y \in m$, that

$$
\langle d \tau(y) f, h\rangle_{\theta}=\langle f, d \tau(y) h\rangle_{\theta} .
$$


It follows that the null space of $\langle,\rangle_{\theta}$ intersection with $\mathscr{S}(V)$ is invariant under $d \tau(y)$ for all $y \in m$. So we may define, on the domain $\mathscr{D}=\varphi(\mathscr{S}(V))$, the operators

$$
d \rho(y)(\varphi(f)):=\varphi(d \tau(y) f), \quad y \in m .
$$

Moreover $d \rho(y)(\mathscr{D}) \subset \mathscr{D}$ for all $y \in m$.

Now let $f \in \mathscr{S}(V)$. Then there is a neighborhood $V_{f}$ of $e \in K$ which satisfy (A5). Let $\varphi(f) \in \mathscr{D}$. Then define $W_{\varphi(f)}:=V_{f}$. Hence $W_{\varphi(f)}$ verifies (LR5) of [J1]. An application of the theorem in [J1] then concludes with the proof.

\section{REFERENCES}

[FOS] J. Fröhlich, K. Osterwalder, and E. Seiler, On virtual representations of symmetric spaces and their analytic continuation, Ann. of Math. 118 (1983), 461-489.

[H] S. Helgason, Differential geometry and symmetric spaces, Academic Press, New York and London, 1962.

[J1] P. E. T. Jorgensen, Analytic continuation of local representation of Lie groups, Pacific J. Math. 125 (1986), 397-408.

[J2] _ Analytic continuation of local representations of symmetric spaces, J. Funct. Anal. 70 (1987), 304-322.

[KL] A. Klein and L. J. Landau, From the Euclidean group to the Poincaré group via OsterwalderSchrader positivity, Comm. Math. Phys. 87 (1983), 469-484.

[LM] M. Lüscher and G. Mack, Global conformal invariance in quantum field theory, Comm. Math. Phys. 41 (1975), 203-234.

[OS] K. Osterwalder and R. Schrader, Axioms for Euclidean Green's functions, Comm. Math. Phys. 31 (1973), 83-112; Part II, 42 (1975), 281-305.

[P1] H. Prado, Local representations of symmetric spaces and O-S positivity, $\mathrm{Ph} . \mathrm{D}$. Thesis, University of Iowa, 1989.

[P2] _ Linear subspaces with analytic structure, and approximation in Hilbert space (to appear).

[S] R. Schrader, Reflection positivity for the complementary series of $\operatorname{SL}(2 n, \mathrm{C})$, Publ. Res. Inst. Math. Sci. 22 (1986), 119-141.

[Se] E. Seiler, Gauge theories as a problem of constructive quantum field theory and statistical mechanics, Lecture Notes in Physics, Springer-Verlag, Berlin, Heidelberg, and New York, 1982.

Department of Mathematics, Oxlahoma State University, Stillwater, Oklahoma 74078

- Current address: Departamento de Matemáticas, Universidad Católica del Norte, Casilla 1280 , Antofagasta, Chile 